\title{
Mechanisms of type I interferon action and its role in infections and diseases transmission in mammals
}

\author{
Paulina Niedźwiedzka-Rystwej ${ }^{1 凶}$, Weronika Ratajczak* ${ }^{*}$ Beata Tokarz-Deptuła ${ }^{1}$ and \\ Wiesław Deptuła ${ }^{2}$
}

'Department of Immunology, Faculty of Biology, University of Szczecin, Szczecin, Poland, 2Department of Microbiology, Faculty of Biology, University of Szczecin, Szczecin, Poland

\begin{abstract}
Interferons (IFNs) are pivotal regulators of immunological processes. This paper describes mainly type I interferons $-\alpha$ and $-\beta$ and their recently recounted signaling pathways, especially connected with ISGs - interferon stimulated genes, having a crucial role in regulating IFN recruitment. Moreover, the paper shows the data on the role of interferons $-\alpha$ and $-\beta$ in infections - not only commonly known viral infections, but also bacterial, fungal and parasitic.
\end{abstract}

Key words: interferon, signaling pathway, infection, mammal

Received: 02 August, 2016; revised: 21 August, 2016; accepted: 21 August, 2016; available on-line: 07 April, 2017

e-mail: kurp13@univ.szczecin.pl

*Student, Scientific Circle of Microbiologists, Faculty of Biology, University of Szczecin

Abbreviations: DAI, DNA-dependent activator of IFN regulatory factor; DC, dendritic cell; iNOS, induced nitric oxide synthase IFN, interferon; IL-1, interleukin 1; IFNAR, interferon $\alpha / \beta$ receptor; IRF3, IFN-regulatory factor 3; ISG, IFN-stimulated genes; JAK, Janus kinase; MAVS, mitochondrial adaptor proteins; MDA-5, melanoma differentiation gene-5; NK, natural killer; NLR, Nod-like receptors; NOD, nucleotide oligomerization domains; PAMP, pathogen associated molecular patterns; PRR, pattern recognition receptors; RLR, RIG-I like receptors; STING, stimulator of interferon genes; STAT, signal transducer and activator of transcription; TBK1, TANK-binding kinase 1; TLR, Toll-like receptors; TRIF, TIR-domain-containing adapter-inducing interferon- $\beta$; TYK2, tyrosine kinase 2

\section{INTRODUCTION}

Response of an organism to infections caused by microorganisms is a highly complex process involving different types of the immune cells and different immunological mechanisms. Distinct elements and mechanisms of the immune response are activated depending on the infectious factor, and these elements are modulated by i.e. cytokines, which significantly influence the course of the immune response, including inflammation. Interferons (IFNs) belong to the group of important regulators of immunological processes.

These cytokines can be divided into three types: type I (IFN-I), type II (IFN-II) and type III (IFN-III) (Alsharifi et al., 2008; Koyama et al., 2008; Gessani et al., 2014; Crouse et al., 2015; McNab et al., 2015). Type I interferons include various variants of $\operatorname{IFN} \alpha$ (13 in humans and 14 in mice) produced by leukocytes (Alsharifi et al., 2008, Gessani et al., 2014; Davidson et al., 2015; McNab et al., 2015). IFN $\beta$ is synthesized by fibroblasts, IFN $\varepsilon$ by cells of the placenta (Durbin et al., 2013; Ivashkiv \& Donlin, 2014;), IFN $x$ by keratinocytes (Durbin et al., 2013), IFN $\omega$ is produced by leukocytes and shows a similar activity to IFN $\alpha$ (Durbin et al., 2013), whereas IFN $\tau$ and IFN $\zeta$ are synthesized and secreted by T and B lymphocytes (Alsharifi et al., 2008, Sadler \& Williams, 2008; Gessani et al., 2014; Davidson et al., 2015; McNab et al., 2015), but their function is still not well known. IFN-II, also known as IFN $\gamma$ is produced mainly by T, NK and NKT cells (Alsharifi et al., 2008, Gessani et al., 2014; Davidson et al., 2015; McNab et al., 2015), and IFN-III comprises IFN $\lambda 1$ (IL-29), IFN $\lambda 2$ (IL-28A), IFN $\lambda 3$ (IL-28B), which have similar functions to IFN-I, but their expression is detected only in epithelial cells (McNab et al., 2015). In the human organism in response to viral, bacterial, fungal as well as parasitic infections the cells produce mainly IFN $\alpha$ and IFN $\beta$ of the type I interferons, thus in this review the term IFN-I will be used to describe these two subtypes of IFN (Alsharifi et al., 2008; Sadler \& Williams, 2008; McNab et al., 2015).

Interferons $-\alpha$ and $-\beta$ play an important role in regulation of the innate immune system, especially modulating the functions of macrophages and dendritic cells. In addition, these cytokines significantly influence the adaptive immune response, regulating the function of $\mathrm{T}$ lymphocytes, mainly CD4+ and CD8+ cells (Gessani et al., 2014; McNab et al., 2015), i.e. by influencing Th lymphocytes polarization, as well as activation of Tc, NK and B cells (Alsharifi et al., 2008; Gessani et al., 2014). IFN-I are also involved in regulation of apoptosis and autophagy - important cellular processes activated in the course of viral and bacterial infections (Trinchieri, 2010; Malireddi et al., 2013; Schmeisser et al., 2014; McNab et al., 2015). Studies showed that IFN- $\alpha$ and $-\beta$ take part in activation of inflammasomes - functional receptors, thus regulating the IL-1 synthesis and indirectly influencing pyroptosis, an inflammatory cell death process dependent on caspase- 1 which is released by inflammasomes interactions (Malireddi et al., 2013; Pothlichet et al., 2013). Both cytokines exert a pleiotropic effect by inducing antiviral immunity in infected and non-infected cells, as well as in the bystander cells, through activation of transcription of genes interacting with the virus replication cycle (McNab et al., 2015). The key factor causing activation of cell signaling pathways that lead to IFN- $\alpha$ and $-\beta$ synthesis is recognition of the pathogen associated molecular patterns (PAMP) by the pattern recognition receptors (PRR). On the other hand both interferons induce signal transduction in the cells after binding to specific cell surface receptors: IFNAR (interferon $\alpha / \beta$ receptor), which leads to activation of transcription of IFN-stimulated genes (ISG), whose protein products play an important role in the immune response (Durbin et al., 2000; Koyama et al., 2008; Sadler \& Williams, 2008; Trinchieri, 2010; Crouse et al., 2015; Durbin et al., 2013; Urban \& Welsh, 2014; McNab et al., 2015). 


\section{IFN- $\alpha$ AND - $\beta$ SIGNALING PATHWAYS}

\section{PRR receptors and IFN- $\alpha$ and $-\beta$ induction}

Effective immune response requires correct identification of the infectious agent by the cell, which is achieved by binding of PRR with PAMP. PRR, which are involved in transcriptional activation of IFN-I and are induced by microorganisms, include: TLR (Toll-like receptors), RLR (RIG-I like receptors, retinoic acid-inducible gene I like receptors), NLR (Nod-like receptors), as well as DAI family receptors (DNA-dependent activator of IFN regulatory factor) and enzymes, such as: DHX36 and DHX9 helicases, RNA polymerase III (Bonjardim, 2005; Alsharifi et al., 2008; Koyama et al., 2008; Trinchieri, 2010; Rathinam \& Fitzgerald, 2011; Swiecki \& Colonna, 2011; Taylor \& Mossman, 2013; Crouse et al., 2015; Urban \& Welsh, 2014; White \& Kile 2015).

TLR3 and TLR4 receptors, which recognize viral genetic material, e.g. dsRNA and bacterial lipopolysaccharides, and regulate type I IFN production, belong to TLRs expressed mainly on macrophages and dendritic cells (DCs) (Bonjardim 2005; Sadler \& Williams, 2008; Trinchieri, 2010; Durbin et al., 2013; Taylor \& Mossman 2013). These receptors bind TRIF (TIR-domain-containing adapter-inducing interferon- $\beta$ ) adaptor molecule initiating the signal transduction and activating TANK-binding kinase1 (TBK1), which is a key enzyme in IFN- $\alpha$ and $-\beta$ production, or $\mathrm{I} \varkappa \mathrm{B}$ kinase- $\varepsilon(\mathrm{IKK} \varepsilon)$ leading to phosphorylation/activation of IRF3 (IFN-regulatory factor 3) transcription factor. This in turn results in transcriptional activation of genes encoding IFN-I (Malmagaard, 2004; Onoguchi et al., 2007; Trinchieri, 2010; Richards \& Macdonald, 2011; Yesebrant et al., 2014; McNab et al., 2015). According to Swiecki and Colonna (Swiecki \& Colonna, 2011) TLR2, expressed by monocytes and DCs, contributes to IFN- $\alpha$ and $-\beta$ production by these cells via recognition of viral hemagglutinin; whereas, stimulation of plasmocytoid dendtritic cells (pDCs), also synthesizing IFN-I in the course of other viral infections, i.e. HIV-1 (Swiecki \& Colonna, 2010; Vermeire et al., 2015), influenza (Killip et al., 2015), Sendai virus or HSV (Swiecki \& Colonna, 2010), is induced by different pathways. In the case of infections with ssRNA viruses $\mathrm{pDCs}$ are activated by endosomal TLR7 (Killip et al., 2015), while ssDNA viruses stimulate TLR9 (Swiecki \& Colonna, 2010; Tang et al., 2010). TLR9 is a surface receptor expressed by pDCs and may also be activated by bacterial DNA (Malmagaard, 2004; Koyama et al., 2008; Lousberg et al., 2010; Trinchieri, 2010; Rathinam \& Fitzgerald, 2011; Swiecki \& Colonna, 2011). TLR7/9 induced signaling pathways are mediated by adaptor protein MyD88 which recruits transcription factor IRF7 (IFN-regulatory factor 7) expressed in lymphoid tissues and activated by the same kinases as IRF3 (Malmagaard, 2004; Bonjardim 2005; Koyama et al., 2008; Trinchieri, 2010; Richards \& Macdonald, 2011; Yan \& Chen 2012; Durbin et al., 2013; Paludan \& Bowie, 2013; Yesebrant de Lendock \& Martinet, 2014).

IFN- $\alpha$ and $-\beta$ synthesis is also regulated by cytoplasmic receptors from RLR family, which consists of three different types of receptors: retinoic acid inducible gene-I (RIG-I), melanoma differentiation gene-5 (MDA5) and laboratory of genetics and physiology-2 (LGP2). RIG-I has an activity of RNA helicase and recognizes viral dsRNA with its helicase domain (Ramos \& Gale, 2011). RIG-I was also shown to be induced by ssRNA of paramyxoviruses, orthomyxoviruses (group A and B), flaviviruses and arboviruses, e.g. Japanese encephalitis virus (JEV) (Onoguchi et al., 2007). Another RLR family member - MDA5 senses dsRNA of rotaviruses, as well as ssRNA of picornaviruses (Koyama et al., 2008; Ramos \& Gale, 2011; Yan \& Chen, 2012; Goubau et al., 2013). Upon recognition of viral RNA these receptors are recruited by mitochondrial adaptor proteins MAVS (also known as IPS-1) or STING (stimulator of interferon genes) - an endoplasmic reticulum associated proteins (Swiecki \& Colonna, 2011; Liu et al., 2015), leading to activation of TBK1/IKKe kinases, which in turn activates transcription factor IFR3 (Koyama et al., 2008; Trinchieri, 2010; Ramos \& Gale, 2011; Durbin et al., 2013; Goubau et al., 2013; Liu et al., 2015).

Nod-like receptors (NLR) divided into four subfamilies (NLRA, NLRB, NLRC, NLRP) also belong to intracellular cytosolic sensors containing nucleotide oligomerization domains (NOD). NLRC: NOD1 and NOD2 contain additionally caspase recruitment domain (CARD) (Trinchieri, 2010), whereas NLRP, such as NLRP3, characterized by their PYRIN domains are involved in formation of inflammasomes and after binding viral ssRNA or dsRNA they lead to caspase-1 activation inducing pyroptosis (Koyama et al., 2008). NOD receptors recognize exogenous nucleic acids and muropeptides of bacterial walls (Trinchieri, 2010), transducing the signal via RICK (receptor-interacting serine-threonine kinase), which leads to induction of $\mathrm{NF} x \mathrm{~B}$ causing cytokines production i.e. during Mycobacterium tuberculosis and Helicobacter pylori infections (Trinchieri, 2010). Furthermore, RICK was shown to interact with TRAF3 and MAVS causing activation of TBK1/IKK $\varepsilon$ kinases and phosphorylation of IRF5 and IRF7 transcription factors involved in regulation of IFN $\beta$ gene expression (Trinchieri, 2010; Durbin et al., 2013). Studies showed that during infection with Newcastle disease virus (NDV) or HSV-1, high level of IRF5 expression was detected mainly in lymphoid tissue, whereas blood cells displayed low expression of this transcription factor (Malmagaard, 2004).

Several other cytosolic DNA sensors, such as: DAI (DNA-dependent activator of IFN regulatory factor) also known as ZBP1 (Z-DNA-binding protein 1) or DLM1, RNA polymerase III, LRRFIP1, DDX36/DHX9 and IFI16 are involved in stimulation of IFN- $\alpha$ and $-\beta$ synthesis in response to intracellular pathogens (Rathinam \& Fitzgerald, 2011; Swiecki \& Colonna, 2011; Yan \& Chen 2012; Goubau et al., 2013; Paludan \& Bowie, 2013; Yesebrant de Lendock \& Martinet, 2014; McNab et al., 2015). DAI receptors recognize viral and bacterial DNA, causing IFN- $\alpha$ and $-\beta$ production via signaling pathways mediated by TBK1/IKKe kinases (Rathinam \& Fitzgerald, 2011; Yesebrant de Lendock \& Martinet, 2014), whereas LRRFIP1 is the viral and bacterial DNA sensor, which induces transcription of IFN $\beta$ gene via pathway involving $\beta$-catenin (Rathinam \& Fitzgerald, 2011; Yesebrant de Lendock \& Martinet, 2014). The mechanism of LRRFIP1 actions was described in regard to infections caused by e.g. Listeria monocytogenes (Rathinam \& Fitzgerald, 2011; Yesebrant de Lendock \& Martinet, 2014). On the other hand IFI16, containing PYHIN domain within its structure binds nucleic acids of pathogens and interacts with STING protein, causing TBK1 activation and induction of IRF3 activity (Ishikawa et al., 2009; Rathinam \& Fitzgerald, 2011; Paludan \& Bowie, 2013; Yesebrant de Lendock \& Martinet, 2014). Studies showed that STING is a key factor in stimulation of IFN $\beta$ synthesis in the course of infections caused by adenoviruses and herpes viruses (HSV1 and HSV2) (Ishikawa et al., 2009). 
DHX36 (DExD/H-box helicase 36) and DHX9 (DExD/H-box helicase 9) also play an important role in IFN- $\alpha$ and $-\beta$ production. These enzymes were shown to recognize $\mathrm{CpG}-\mathrm{A}$ and $\mathrm{CpG}-\mathrm{B}$ DNA of HSV viruses in the cytosol of pDCs. Furthermore, RNA polymerase III is another enzyme involved in regulation of IFN-I synthesis. RNA polymerase III senses AT-rich DNA of adenoviruses or Epstein-Barr gamma herpes virus, and transcribes it into immunostimulatory RNA transcripts, which stimulate RIG-I receptors (Rathinam \& Fitzgerald, 2011; Paludan \& Bowie, 2013).

In addition, mitochondria of infected cells are also involved in the mechanisms of IFN- $\alpha$ and $-\beta$ production (White \& Kile, 2015). In stress conditions caused by infection mitochondrial DNA (mtDNA) is released from these organelles and binds to cyclic GMP-AMP synthase (cGAS). The mtDNA/cGAS complex activates STING, which in turn induces transcription of IFN- $\alpha$ and $-\beta$ genes via signaling pathway mediated by TBK1 kinase and IRF3 transcription factor (White \& Kile, 2015).

\section{ISG - interferon stimulated genes}

IFN- $\alpha$ and $-\beta$, which are synthesized in response to receptors activation and signal transduction, leading to stimulation of IFN-I genes expression, are responsible for induction of interferon stimulated genes - ISG. In the classical ISG-activation pathway IFN- $\alpha$ and $-\beta$ bind to their transmembrane receptor that consists of two subunits: IFNAR1 (interferon $\alpha / \beta$ receptor 1 ) and IFNAR2 (interferon $\alpha / \beta$ receptor 2 ) (Bonjardim 2005; Ho \& Ivashkin, 2006; Lousberg et al., 2010; Gonzales-Navajas et al., 2012; Durbin et al., 2013; Ivashkiv \& Donlin, 2014; Levin et al., 2014; Davidson et al., 2015; McNab et al., 2015). This leads to activation of JAK tyrosine kinases associated with these receptor subunits, namely JAK1 (Janus kinase 1) and TYK2 (tyrosine kinase 2), which in turn phosphorylate their downstream effectors - transcription factors STAT1 and STAT2 (signal tranducers and activators of transcription 1 and 2) (Durbin et al., 2000; Bonjardim 2005; Casanova et al., 2012; Rauch et al., 2013, Ivashkiv \& Donlin, 2014; Levin et al., 2014; Davidson et al., 2015; McNab et al., 2015), causing their dimerisation and interaction with IRF9. As a result the ISG factor 3 (ISGF3) complex is formed, translocates into the nucleus and binds to IFN-stimulated regulatory elements (ISREs) within the promoter region of ISG (Bonjardim 2005; Ho \& Ivashkin, 2006; Casanova et al., 2012; Gonzales-Navajas et al., 2012; Durbin et al., 2013; Ivashkiv \& Donlin, 2014; Levin et al., 2014; Davidson et al., 2015; McNab et al., 2015). ISREs have a characteristic sequence 'TTTCNNTT'TC (Malmagaard, 2004; Ivashkiv \& Donlin, 2014), and recognition of this site by ISGF3 leads to transcription activation of even hundreds of ISGs (Richards \& Macdonald, 2011; Ivashkiv \& Donlin, 2014; Levin et al., 2014; McNab et al., 2015; Schmeisser et al., 2014). Currently, ISGs can be divided into two groups: "robust genes", activated even by low concentrations of weak-binding IFNs, and "tunable genes" whose activation requires a high concentration of high-affinity IFNs as well as high concentration of surface receptors. Products of robust genes show antiviral activity, whereas tunable genes encode proteins showing chemokine activity, and regulating cell proliferation or inflammatory response (Levin et al., 2014). ISG activation may be induced by classical or alternative signaling pathways that differ only in the type of transcription factors involved in stimulation of their transcription. The abovementioned classical pathway involves kinases: JAK1,
JAK2, TYK2 (tyrosine kinase), whereas alternative route of ISG activation is mediated by transcription factors from STAT family: STAT3, STAT4, STAT5A, STAT5B, which also may be induced by signaling pathways activated by other cytokines (Casanova et al., 2012; McNab et al., 2015). Thus, the pleiotropic effects of IFN- $\alpha$ and $-\beta$ result from their ability to induce various routes of activation of a broad spectrum of ISGs, whose protein products are directly involved in restriction of viral replication, inhibition of bacterial growth, but also may regulate synthesis of other cytokines and chemokines modulating the functions of the immune cells, or acting as pro-apoptotic factors (Lopez et al., 2006; Ivashkiv \& Donlin, 2014; Levin et al., 2014; McNab et al., 2015). Studies showed that IFNs-induced signaling pathway mediated by STAT3/STAT5 has anti-apoptotic and promitogenic effects, while STAT4-mediated signaling promotes IFN $\gamma$ synthesis and clonal expansion of lymphocytes (Urban \& Welsh, 2014).

\section{IFN- $\alpha$ AND $-\beta$ IN VIRAL INFECTIONS}

It was well documented that activation of ISG in infected and neighboring cells results in the synthesis of factors limiting viral replication, i.e. RNA-activated protein kinase $\mathrm{R}$ (PKR) and 2'-5'-oligoadenylate synthetase (OAS) (Bonjardim 2005; Alsharifi et al., 2008, Koyama et al., 2008; Sadler \& Williams, 2008; Davidson et al., 2015; Durbin et al., 2013; Ivashkiv \& Donlin, 2014; Levin et al., 2014; McNab et al., 2015). Furthermore, this process leads to activation of $\mathrm{Mx}$ protein (Sadler \& Williams, 2008; Yan \& Chen 2012; Levin et al., 2014; Sandler et al., 2014), apolipoprotein APOBEC (Bonjardim 2005; Yan \& Chen, 2012; Schmeisser et al., 2014; McNab et al.,2015) and interferon-induced transmembrane proteins IFITM (Bonjardim 2005; Yan \& Chen 2012; McNab et al., 2015). These factors also inhibit the translational apparatus or induce intracellular degradation of ssRNA viruses in order to limit their spreading (Davidson et al., 2015). Indirect and direct function of IFN- $\alpha$ and $-\beta$ exerts direct or indirect influence on the antigen-presenting cells (APC), such as: DCs, T lymphocytes, NK cells, $\mathrm{B}$ lymphocytes and cells of the myeloid lineage. Studies showed that IFN $\beta$ expression occurs in all virus-infected cells, whereas IFN $\alpha$ is expressed only in APC, especially in pDCs (Crouse et al., 2015; McNab et al., 2015). Furthermore, IFN- $\alpha$ and $-\beta$ are involved in activation of apoptosis and autophagy in cells during viral infections. In addition, recent studies demonstrated that both IFNs may play a role in alternative mechanism of NLRP3 inflammasome activation resulting in the induction of pyroptosis (Malireddi et al., 2013).

$\mathrm{IFN} \alpha$ plays the key role in activation of many immunological factors involved in viral infections and differentiation of macrophages into DCs. This cytokine also takes part in APC maturation and stimulates the expression of MHC class I and II, other surface proteins, e.g.: CD40, CD80, CD83, CD86 (Ou et al., 2001; Lopez et al., 2006; Swiecki \& Colonna, 2010; Swiecki \& Colonna, 2011; Durbin et al., 2013; Crouse et al., 2015; Davidson et al., 2015; Gessani et al., 2014; McNab et al., 2015), chemokine receptors CCR5 and CCR7 and lymphocyte-associated antigen 1 (LFA1) (Durbin et al., 2013; Gessani et al., 2014; Crouse et al., 2015; Davidson et al., 2015; McNab et al., 2015). Therefore, IFN $\alpha$ is often described as "endogenous adjuvant" (Lopez et al., 2006). Moreover, IFN $\alpha$ by acting on DCs regulates chemotaxins production, e.g.: CXCL9 and CXCL10 (CXC-chemo- 
kine ligands) specifically targeting antigen-presenting $\mathrm{T}$ lymphocytes (Crouse et al., 2015; Davidson et al., 2015; $\mathrm{McNab}$ et al., 2015). It was also shown that IFN- $\alpha$ and - $\beta$ indirectly activate $T$ cells via DCs which have the ability to present antigen, migrate and express cytokines stimulating maturation and differentiation of $\mathrm{T}$ lymphocytes population in peripheral lymphatic organs (Crouse et al., 2015; McNab et al., 2015). This mechanism constitutes an important element of antiviral immune response (Swiecki \& Colonna, 2010; Crouse et al., 2015; Hastings et al., 2015).

Another example of indirect effect of IFN-I on T lymphocytes differentiation is the IFN-I- stimulated production and secretion of cytokines by DCs, e.g. IFN $\gamma$, which is involved in activation of Th1 lymphocytes differentiation. In addition, DCs activated by IFN $\alpha$ synthesize IL-15 and IL-7, whose actions induce proliferation and survival of T and NK cells. Stimulation of DCs by IFN- $\alpha$ and $-\beta$ also results in production of IL-12 regulating Th1 cells. On the other hand high concentrations of IFN- $\alpha$ and $-\beta$ may inhibit the activity of Th1 lymphocytes (Lopez et al., 2006; Swiecki \& Colonna M, 2011; Crouse et al., 2015). Studies showed that during influenza virus and cowpox virus infections IFN- $\alpha$ and - $\beta$ cause increased activation of NK cells and stimulate the synthesis of IFN $\gamma$ via pathways mediated by STAT transcription factors, leading to regulation of Th1 cells function (Crouse et al., 2015; McNab et al., 2015). Aside from the indirect effect of IFN- $\alpha$ and $-\beta$ on T lymphocytes, these cytokines may directly influence the functions of CD4+ and CD8+ T cells (Crouse et al., 2015; $\mathrm{McNab}$ et al., 2015). In the case of CD4+ T cells type I IFNs induce their differentiation into Th1 lymphocytes, which in turn synthesize IFN $\gamma$ (Bonjardim 2005; Alsharifi et al., 2008; Swiecki \& Colonna, 2010). IFN- $\alpha$ and $-\beta$ also enhance the immune response of Th1 cells by inhibiting production of cytokines typical for Th2 cells, i.e. IL-4 and IL-5 (Alsharifi et al., 2008). CD4+ T lymphocytes stimulated by IFN $-\alpha$ and $-\beta$ interact with $B$ cells and are involved in their clonal expansion ( $\mathrm{McNab}$ et al., 2015). On the other hand both interferons inhibit growth of CD8+ T lymphocytes through STAT1-mediated signaling pathway (McNab et al., 2015), although despite the antiproliferative function they may regulate CD8+ T cells survival and clonal expansion (Alsharifi et al., 2008; Urban \& Welsh, 2014; Yesebrant de Lendock \& Martinet, 2014; McNab et al., 2015). IFN- $\alpha$ and $-\beta$ are also involved in regulation of differentiation, function and number of memory $\mathrm{T}$ cells (Tm) by inducing their expansion to the sites of viral infection (Alsharifi et al., 2008, Swiecki \& Colonna, 2010; McNab et al., 2015; Urban \& Welsh, 2014). Both interferons act positively on Tm lymphocytes function also in the case of secondary viral infections. It was demonstrated that in the course of Sendai virus infections IFN-I enhance the cytotoxic effect of Tm cells. During the immune response to lymphocytic choriomeningitis virus (LCMV) infections these cytokines induce chemokines production by Tm cells, whereas in mouse cytomegalovirus (MCMV) infections IFN- $\alpha$ and $-\beta$ regulate IL-15 and IL-18 production exerting their effect on monocytes (Alsharifi et al., 2008, McNab et al., 2015). A significant role of IFN- $\alpha$ and $-\beta$ was also observed during chronic LCMV infections (Ou et al., 2001). Studies using a mouse model of arenavirus infections, showed that in the course of long-term infections the cells lost their antigen-specific activity, causing repression or loss of $\mathrm{CD} 8+\mathrm{T}$ lymphocytes, which are specific for this type of infections (Ou et al., 2001; Urban \& Welsh, 2014). Some scientific reports also demon- strated the influence of IFN- $\alpha$ and $-\beta$ on regulatory $T$ cells (Treg) population, causing either a decline in the number of Tregs or induction of these cells proliferation (Hastings et al., 2015).

IFN $-\alpha$ and $-\beta$ are also important regulators of $\mathrm{NK}$ cells function, increasing their proliferation, maturation as well as accelerating the cytotoxic activity of these cells and IFN $\gamma$ synthesis (Stackaruk et al., 2013). Moreover, it was shown that activation of the cytotoxic activity of NK cells by type I IFNs (Ou et al., 2001; Swiecki \& Colonna, 2010; Swiecki \& Colonna, 2011; Chijioke \& Munz 2013; Crouse et al., 2015) stimulates the pDCs to produce IFN- $\alpha$ and $-\beta$ (Chijioke \& Munz, 2013).

In addition, IFN $-\alpha$ and $-\beta$ stimulate B lymphocytes to become antibodies producing B cells (Lopez et al., 2006; Swiecki \& Colonna, 2011), although these cytokines may also decrease survival and development of the precursor and immature B cells (Alsharifi et al., 2008, Yesebrant de Lendock \& Martinet, 2014). During viral infections IFN- $\alpha$ and $-\beta$ induce the secretion of $B$ lymphocyte stimulator (BLyS, also known as BAFF) and A proliferation-inducing ligand (APRIL) by activation of macrophages and DCs (Kiefer et al., 2012). These proteins constitute the key factors for the survival of B cells in the periphery (Kiefer et al., 2012). The positive role of both type I IFNs in activation of B lymphocytes is also connected with their ability to regulate the profile of IgG antibodies subclasses synthesized by these cells in the course of influenza virus infections (Alsharifi et al., 2008, Kiefer et al., 2012; McNab et al., 2015). Furthermore, IFN- $\alpha$ and $-\beta$ induce production of IgM and IgA antibodies by B cells (Alsharifi et al., 2008; Swiecki \& Colonna, 2010) and are required for activation of B lymphocytes in the lymph nodes, where they are additionally involved in production of TNF $\beta$ - a cytokine showing protective function towards a specific phenotype of macrophages (McNab et al., 2015). It was demonstrated that during infection of human macrophages with avian influenza virus subtype H5N1 type I interferons, especially IFN $\beta$, are among the cytokines appearing at the earliest stages of infection (Moulin et al., 2011; Davidson et al., 2015), and can be detected before other proinflammatory cytokines and chemokines, such as: IL-12 and macrophage inflammatory protein $1 \beta$ (Mip-1 $\beta$ ) (Davidson et al., 2015). Increased level of IFN $\alpha$ during $\mathrm{H} 5 \mathrm{~N} 1$ virus infection is connected with augmented secretion of cytokines causing aberrations in coagulation, which was also demonstrated in the course of viral haemorrhagic fevers (Moulin et al., 2011).

IFN $-\alpha$ and $-\beta$ not only play an important role in the regulation of the immune cells functions, but also are involved in the regulation of cellular mechanisms leading to different types of programmed cells death - caspase dependent apoptosis (Koyama et al., 2008; Davidson et al., 2015) as well as autophagy (Trinchieri, 2010; Levine et al., 2011; Durbin et al., 2013), which are activated during viral infections. Apoptosis induction requires activation of specific cell signaling pathways initialized by binding of ligands to specific surface membrane receptors. Ligands known to induce this process include cytokines, such as Apo2L protein also known as TRAIL (TNF-related apoptosis - inducing ligand), which belongs to the TNF superfamily (TNFSF10). TRAIL binds to death receptor 5 (DR5) on the cell membrane activating the extrinsic programmed death pathway. During influenza A virus infections increased levels of IFN- $\alpha$ and $-\beta$ result in an increase in TRAIL expression in monocytes and accelerated expression of death receptors on infected epithelial cells, causing host's inflammatory re- 
sponse (Davidson et al., 2015; McNab et al., 2015). Increase in TRAIL and DR5 expression was also noted in the course of HIV-1 infection in pDCs (McNab et al., 2015). In addition, IFN- $\alpha$ and $-\beta$ were shown to induce the extrinsic apoptotic pathway by regulating expression of FAS (CD95), which binds to another apoptosis inducer - FAS ligand (Crouse et al., 2015). Moreover, this programmed cell death may be induced in virus-infected macrophages via TLR4-dependent mechanisms involving synthesis of PKR regulated by ISG expression (Sadler \& Williams, 2008).

On the other hand, autophagy was shown to be required for the production of IFN $\alpha$ by pDCs following recognition of viral antigens by TLR7 (Lee et al., 2007; Lee \& Iwasaki, 2008; Swiecki \& Colonna, 2010; Levine et al., 2011; Schmeisser et al., 2014). Some studies demonstrated negative regulation of RLR by IFN- $\alpha$ and $-\beta$, resulting from interactions of ATG (autophagy related proteins) proteins: ATG5-ATG12 involved in autophagy induction, with RIG-I domains of these receptors (Levine et al., 2011). Additionally, ATG9 was shown to negatively regulate STING (Levine et al., 2011). Other studies describe the role of IFN- $\alpha$ and $-\beta$ in autophagy induction through the classic activation pathway mediated by IFNAR receptor, JAK and TYK kinases and STAT transcription factors (Schmeisser et al., 2014). Moreover, IFN- $\alpha$ and $-\beta$ were shown to activate PI3K (phosphoinositide 3-kinase) pathway, which is known to negatively regulate autophagy via activation of mTORC1 (mammalian target of rapamycin complex 1) and phosphorylation of ATG proteins (Kudchodkar \& Levine, 2009; Durbin et al., 2013; Schmeisser et al., 2014). Simultaneously, mTOR kinase activation by IFNI controls cell growth and metabolism during infections with several viruses, including Epstein-Barr virus, Kaposi sarcoma-associated herpesvirus (KSHV), hepatitis C virus (HCV), human papillomavirus (HPV16) and retroviruses (Trinchieri, 2010; Levine et al., 2011; Durbin et al., 2013). This leads to autophagy inhibition and tumorigenesis promotion (Trinchieri, 2010; Levine et al., 2011). TBK1 constitutes another link between IFN-I and autophagy, as this enzyme is involved in activation of the complex stimulating ISG transcription, as well as regulates autophagy, which is a cellular process subjected to viral suppressive mechanisms, leading to increase in ISG expression in infected cells (Zhao, 2013). Furthermore, studies demonstrated that during infection with influenza virus, there is an accelerated production of IFN- $\alpha$ and $-\beta$ resulting in limitation of the infection by induction of IL- 5 and IL-10 secretion (Durbin et al., 2000).

\section{IFN- $\alpha$ AND - $\beta$ IN BACTERIAL INFECTIONS}

It is commonly accepted that IFN- $\alpha$ and $-\beta$ play a role in bacterial infections; however, detailed mechanisms of their actions have not been fully elucidated yet. Studies demonstrated that in the course of Listeria (L.) monocytogenes infection secretion of hemolytic toxin - listeriolysin O (LLO) causes increase in IFN-I production in macrophages via RLR- and STING-dependent pathways (Malireddi et al., 2013). The signal is then mediated by TBK1-IRF3 axis (Swiecki \& Colonna, 2011; Malireddi et al., 2013), resulting in the synthesis of highly toxic nitric oxide (NO) (Rauch et al., 2013). Furthermore, L. monocytogenes triggers assembly of inflammasomes, such as AIM2, NLRC4 and NLRP3, and this process, connected with pyroptosis activation, is regulated by IFN- $\alpha$ and $-\beta$ (Gonzales-Navajas et al., 2012; Malireddi et al., 2013; Pothlichet et al., 2013; Rauch et al., 2013). Type I interferons are also involved in STAT1-dependent induction of apoptosis during $L$. monocytogenes infection ( $\mathrm{McNab}$ et al., $2015)$. It was shown that IFN- $\alpha$ and $-\beta$ protect macrophages and lung epithelial cells infected with Legionella (L.) pneumophila through induction of MAVS and IRF3 pathway (Gonzales-Navajas et al., 2012). In addition, these cytokines were shown to induce cell death processes, including apoptosis and pyroptosis during $L$. pneumophila infections most probably by upregulation of pro-cell death molecules, such as BAK (BCL2-antagonist/killer 1) and TRAIL (Malireddi et al., 2013). Similar protective mechanism of IFN-I actions was demonstrated in the course of Bacillus anthracis infections. IFN- $\alpha$ and $-\beta$ were reported to inhibit germination of $B$. anthracis spores (Malireddi et al., 2013; McNab et al., 2015). Francisella (F.) tularensis and F. tularensis subsp. novicida also belong to intracellular bacteria causing induction of IFN- $\alpha$ and $-\beta$ secretion in an IRF3-dependent manner, which leads to formation of AIM2 inflammasome (Gonzales-Navajas et al., 2012; Malireddi et al., 2013; Pothlichet et al., 2013; McNab et al., 2015). During Salmonella typhimurium infections type I IFNs induce STAT4-dependent synthesis of IFN $\gamma$ (Trinchieri, 2010) accelerating the cell death processes (Malireddi et al., 2013). On the other hand, during Chlamydia infections type I interferons inhibit the pathogen's growth cycle at the point of transformation of elementary body (EB) into reticulate body (RB), resulting in inhibition of Chlamydia replication (Trinchieri, 2010). It was suggested that in the case of infections with Chlamydia pneumopniae IFN- $\alpha$ and $-\beta$ interact with IFN $\gamma$, allowing the host to effectively limit the survival of the pathogen (McNab et al., 2015). Studies conducted on mouse models showed that IFN- $\alpha$ and $-\beta$ have protective role during Streptoccocus (S.) pneumoniae, S. pyogenes, Pseudomonas aeruginosa, Helicobacter pylori and Echerichia coli infections (Swiecki \& Colonna, 2011; Malireddi et al., 2013). However, the activity of IFN-I not always brings a positive outcome for the infected organism. It was demonstrated that in the case of infections with Mycobacterium (M.) $s p$. elevated expression and secretion of IFN- $\alpha$ and $-\beta$ lead to increased $M$. tuberculosis virulence and suppress the production of proinflammatory cytokines IL- $1 \alpha$ and IL-1 $\beta$, leading additionally to inhibition of IL$1 \beta$ secretion (Ivashkiv \& Donlin, 2014). Such effect can be caused by repression of the activity of NLRP 1 and NLRP3 inflammasomes in a STAT1-dependent manner, thereby inhibiting IL-1 $\beta$ production (Guarda et al., 2011; Gonzales-Navajas et al., 2012; Malireddi et al., 2013; McNab et al., 2015). Experimental mouse models were also used to study the effect of IFN- $\alpha$ and $-\beta$ on the immune system in the presence or absence of the commensal bacteria (Rauch et al., 2013; Ivashkiv \& Donlin, 2014; McNab et al., 2015). Lack of functional intestinal microflora, caused i.e. by antibiotic treatment, results in strong reduction of ISG expression. Moreover, in the absence of IFNAR signaling in the intestinal epithelial cells there is an increase in Paneth cells proliferation, leading to changes in the intestinal microflora. Studies showed that the commensal microbial flora of the intestines determines the basal level of IFN- $\alpha$ and $-\beta$ production, providing the protective function and maintaining organism in homeostasis upon response to pathogenic factors (Ivashkiv \& Donlin, 2014; McNab et al., 2015). 


\section{IFN- $\alpha$ AND - $\beta$ IN FUNGAL AND PARASITIC INFECTIONS}

The role of IFN-I was also indicated in fungal infections. During infections with Candida (C.) albicans, Cryptococcus neoformans IFN- $\alpha$ and $-\beta$ were shown to be involved in induction of reactive oxygen species (ROS) formation, enabling $C$. albicans elimination in the course of phagocytosis. In the case of Cryptococcus neoformans infections both interferons maintain the immune response of the organism by sustaining high levels of IFN $\gamma$, TNF, induced nitric oxide synthase (iNOS) and CXCL10 chemokine (McNab et al., 2015). The role of IFN- $\alpha$ and $-\beta$ was also demonstrated during Candida glabrata and Histoplasma capsulatum infections, although the exact mechanisms of their action have not been elucidated so far (Malireddi et al., 2013; McNab et al., 2015). Nevertheless, it was documented that different forms of fungal glucans and mannans are recognized by TLR and CLR receptors activating signaling pathways which lead to cell death induction or cytokines synthesis (Malireddi et al., 2013).

The immune response of the organism is also regulated by IFN- $\alpha$ and $-\beta$ during Leishmania (L.) major, Plasmodium (P) spp. and Trypanosoma cruzi infections. Studies demonstrated that type I IFNs induce iNOS during leishmaniasis, although too high levels of these cytokines may result in weakened iNOS induction (McNab et al., 2015; Paludan \& Bowie, 2013). Furthermore, high activity of iNOS may suppress the function of macrophages, as well as formation of neutrophils and their number. This dual effect of IFN-I on the immune response was also noted in Plasmodium infections. In the case of $P$. berghei and $P$. chabaudi infections IFN- $\alpha$ and $-\beta$ may augment the parasitic invasion suppressing the function of CD4+ T cells; whereas, during $P$. yoelii infections these cytokines exert a positive effect causing reticulocytosis inhibition (McNab et al., 2015) Similar results were obtained in the studies on Trypanosoma infections, as these parasites may regulate $\mathrm{NO}$ synthesis and negatively affect $\mathrm{T}$ cells producing IFN $\gamma$, the cytokine playing an important role during Trypanosoma cruzi infections ( $\mathrm{McNab}$ et al., 2015).

\section{SUMMARY}

Despite the fact that the functions of IFN- $\alpha$ and $-\beta$ are often described as non- immunological, their role in the immune response during viral, bacterial, fungal and parasitic infections is significant. IFN-I actions are mediated by PRR receptors expressed on the surface of the immune cells, and result in induction of cell death process, i.e. apoptosis, autophagy and pyroptosis. Furthermore, the expression of IFN- $\alpha$ and $-\beta$ in the immune cells is tightly regulated by specific signaling pathways.

\section{REFERECES}

Alsharifi M, Mullbacher A, Regner M (2008) Inteferon type I responses in primary and secondary infections. Immunol Cell Biol 86: 239245. https://doi.org/10.1038/sj.icb.7100159

Bonjardim CA (2005) Interferons (IFNs) are key cytokines in both innate and adaptative antiviral immune responses and viruses counteract IFN action. Microbes Infect 7: 569-578. Casanova JL, Holland SM, Notarangelo LD (2012) Inborn errors of human JAKs and STATs. Immunity 36: 515-528. https://doi.org/10.1016/j. micinf.2005.02.001

Chijioke O, Munz C (2013) Dendritic cell derived cytokines in human natural killer cell differentiation and activation. Front Immunol 4: 1-7. https://doi.org/10.3389/fimmu.2013.00365

Crouse J, Kalinke U, Oxenius A (2015) Regulation of antiviral T cell responses by type I interferons. Nat Rev Immuonol 15: 231243. https://doi.org/10.1038/nri3806
Davidson S, Maini MK, Wack A (2015) Disease - promoting effects of type I interferons in viral, bacterial and coinfections. I Interferon Cytokine Res 35: 252-264. https://doi.org/10.1089/jir.2014.0227

Durbin JE, Fernandes-Sesma A, Lee CK, Rao D, Frey AB, Moran TM, Vukmanovic S, Garcia-Sastre A, Levy DE (2000) Type I IFN modulates innate and specific antiviral immunity. J Immunol 164: 4220-4228. https://doi.org/10.4049/jimmunol.164.8.4220

Durbin RK, Kotenko SV, Durbin JE (2013) Interferon induction and function at the mucosal surface. Immunol Rev 255: 25-39. https:// doi.org/10.1111/imr.12101

Gessani S, Conti L, Del Corno M, Belardelli F (2014) Type I interferons as regulators of human antigen presenting cell functions. Toxins 6: 1696-1723. https://doi.org/10.3390/toxins6061696

Gonzales-Navajas JM, Lee J, David M, Raz E (2012) Immunomodulatory functions of type I interferons. Nat Rev Immunol 12: 125-135. https://doi.org/10.1038/nri3133

Goubau D, Deddouche S, Reis e Sousa C (2013) Cytosolic sensing of viruses. Immunity 28: 855-869. https://doi.org/10.1016/j.immuni.2013.05.007

Guarda G, Braun M, Staehli F, Tardivel A, Mattmann C, Foster I, Farlik M, Decker T, Du Pasqquier RA, Romero P, Tschopp J (2011) Type I interferon inhibits interleukin -1 production and inflammasome activation. Immunity 34: 213-223. https://doi.org/10.1016/j. immuni.2011.02.006

Hastings AK, Erickson JJ, Schuster JE, Boyd K.L, Tollefson SJ, Johanson M, Gilchuk P, Joyce S, Williams JV (2015) Role of type I interferon signaling in human metapneumovirus pathogenesis and control of viral replication. J Virol 98: 4405-420. https://doi. org/10.1128/JVI.03275-14

Ho HH, Ivashkin LB (2006) Role of STAT3 in type I interferon responses. Negative regulation of STAT1 - dependent inflammatory gene activation. J Biol Chem 281: 14111-14118. https://doi. org/10.1074/jbc.M511797200

Ishikawa H, Ma Z, Barber GN (2009) STING regulates intracellular DNA - mediated, type I interferon - dependent innate immunity. Nature 461: 788-792. https://doi.org/10.1038/nature08476

Ivashkiv LB, Donlin LT (2014) Regulation of type I interferon responses. Nat Rev Immunol 14: 36-49. https://doi.org/10.1038/ nri3581

Kiefer K, Oropallo MA, Cancro MP, Marshak-Rothstein A (2012) Role of type I interferons in the activation of autoreactive B cells. Immunol Cell Biol 90: 498-504. https://doi.org/10.1038/icb.2012.10

Killip MJ, Fodor E, Randall RE (2015) Influenza virus activation of the interferon system. Virus Res 209: 11-22. https://doi. org/10.1016/j.virusres.2015.02.003

Koyama S, Ishii KJ, Coban C, Akira S (2008) Innate immune response to viral infection. Cytokine 43: 336-341. https://doi. org/10.1016/j.cyto.2008.07.009

Kudchodkar SB, Levine B (2009) Viruses and autophagy. Rev Med Virol 19: 359-378. https://doi.org/10.1002/rmv.630

Lee HK, Lund JM, Ramanthan B, Mizushima N, Iwasaki A (2007) Autophagy - dependent viral recognition by plasmocytoid dendritic cells. Science 315: 1398-1401. https://doi.org/10.1126/science. 1136880

Lee HK, Iwasaki A (2008) Autophagy and antiviral immunity. Curr Opin Immunol 20: 23-29. https://doi.org/10.1016/j.coi.2008.01.001

Levin D, Schneider WM, Hoffmann HH, Yarden G, Busetto AG, Manor O, Sharma N, Rice CM, Schreiber G (2014) Multifaced activities of type I interferon are revealed by s receptor antagonist. Sci Signal. https://doi.org/10.1126/scisignal.2004998

Levine B, Mizushima N, Virgin HW (2011) Autophagy in immunity and inflammation. Nature 469: 323-335. https://doi.org/10.1038/ nature 09782

Liu X, Wang Q, Pan Y, Wang C (2015) Sensing and responding to cytosolic viruses invasion: an orchestra of kaleidoscopic ubiquitinations. Cytokine Growth Factor Rev 26: 379-387. https://doi. org/10.1016/j.cytogfr.2015.03.001

Lopez CB, Yount JS, Hermesh T, Moran TM (2006) Sendai virus infection efficient adaptive immunity independence of type I interferons. J Virol 80: 4538-4545. https://doi.org/10.1128/JVI.80.9.45384545.2006

Lousberg EL, Fraser CK, Tovey MG, Diener KR, Hayball JD (2010) Type I interferons mediate the innate cytokine response to recombinant fowlpox virus but not induction of plasmocytoid dendritic cell - dependent adaptive immunity. J Vorol 84: 6549-6563. https://doi.org/10.1128/JVI.02618-09

Malireddi RKS, Kanneganti TD (2013) Role of type I inflammasome activation, cell death, and disease during microbial infection. Front Cell Infect Microbiol 3: 1-11. https://doi.org/10.3389/ fcimb.2013.00077

Malmagaard L (2004) Induction and regulation of IFN during viral infections. J Interferon Cytokine Res 24: 439-454. https://doi. org/10.1089/1079990041689665

McNab F, Mayer-Barber K, Sher A, Wack A, O'GArra A (2015) Type I interferons in infectious disease. Nat Rev Immunol 15: 87-103. https://doi.org/10.1038/nri3787 
Moulin HR, Liniger M, Python S, Guzylack-Piriou L, Ocana-Macchi M, Ruggi N, Summerfield A (2011) High interferon type I responses in the lung, plasma and spleen during highly pathogenic H5N1 infection of chicken. Vet Res 42: 1-6. https://doi.org/10.1186/12979716-42-6

Onoguchi K, Yoneyama M, Takemura A, Akira S, Taniguchi T, Namiki H, Fujita T (2007) Viral infections activate Tees I and III interferon genes through a common mechanism. I Biol Chem 282 7576-7581. https://doi.org/10.1074/jbc.M608618200

Ou R, Zhou S, Huang L, Moskophidis D (2001) Critical role for alpha/beta and gamma interferons in persistence of lymphocytic choriomeningitis virus by clonal exhaustion of cytotoxic $\mathrm{T}$ cell. J Virol 75: 8407-8423. https://doi.org/10.1128/JVI.75.18.84078423.2001

Paludan SR, Bowie AG (2013) Immune sensing of DNA. Immunity 38: 870-880. http://doi.org/10.1016/j.immuni.2013.05.004

Pothlichet J, Meunier I, Davis BK, Ting JPY, Skamene E, Von Messling V, Vidal SM (2013) Type I IFN triggers RIG-I/TLR3/ NLRP3 - dependent inflammasome activation in influenza A virus infected cells. PLoS Pathog 9: 1-14. https://doi.org/10.1371/journal. ppat.1003256

Ramos HJ, Gale M Jr (2011) RIG - I like receptors and their signaling cross talk in the regulation of antiviral immunity. Curr Opin Virol 1: 167-176. https://doi.org/10.1016/j.coviro.2011.04.004

Rathinam VA, Fitzgerald KA (2011) Innate immune sensing of DNA viruses. Virology 411: 153-162. https://doi.org/10.1016/j.virol.2011.02.003

Rauch I, Muller M, Decker T (2013) The regulation of inflammation by interferons and their STATs. JAKSTAT 2: 1-13. https://doi. org/10.4161/jkst.23820

Richards KH, Macdonald A (2011) Putting the brakes on the antiviral response: negative regulators of type I interferons (IFN) production. Microbes Infect 13: 291-302. https://doi.org/10.1016/j. micinf.2010.12.007

Sadler AJ, Williams BRG (2008) Interferon - inducible antiviral effectors. Nat Rev Immunol 8: 559-568. https://doi.org/10.1038/nri2314

Sandler NG, Bosinger SE, Estes JD, Zhu RTR, Tharp GK, Boritz E, Levin D, Wijeyesinghe S, Makamdop KN, del Prete GQ, Hill BJ, Timmer JK, Reiss E, Yarden G, Darko S, Contijoch E, Todd JP, Silvestri G, Nason M, Norgren RB Jr, Keele BF, Rao S, Langer JA, Lifson JD, Schreiber G, Douek DC (2014) Type I interferon response in rhesus macaques prevent SIV infection and slow dis- ease progression. Nature 511: 601-605. https://doi.org/10.1038/ nature 13554

Schmeisser H, Bekisz J, Zoon KC, (2014) New function of type I IFN: induction of autophagy. J Interferon Cytokine Res 34: 71-78. https:// doi.org/10.1089/jir.2013.0128

Stackaruk ML, Lee AJ, Ashkar AA (2013) Type I interferon regulation of natural killer cell function in primary and secondary infections. Expert Rev Vaccines 12: 875-884. https://doi.org/10.1586/14760584 .2013 .814871

Swiecki M, Colonna M (2010) Unraveling the functions of plasmocytoid dendritic cells during viral infections, autoimmunity and tolerance. Immunol Rev 234: 142-162. https://doi.org/10.1111/j.01052896.2009.00881.x

Swiecki M, Colonna M (2011) Type I interferons: diversity of sources, production pathways and effects on immune responses. Curr Opin Virol 1: 463-475. https://doi.org/10.1016/j.coviro.2011.10.026

Tang F, Du Q, Liu YJ (2010) Plasmocytoid dendritic cells in antiviral immunity and autoimmunity. Sci China Life Sci 53: 172-182. https:// doi.org/10.1007/s11427-010-0045-0

Taylor KE, Mossman KL (2013) Recent advances in understanding viral evasion of type I interferon. Immunology 138: 190-197. https://doi.org/10.1111/imm.12038

Trinchieri G (2010) Type I interferon: friend or foe? J Exp Med 207: 2053-2063. https://doi.org/10.1084/jem.20101664

Urban SL, Welsh RM (2014) Out-of-sequence signal 3 as a mechanism for virus-induced immune suppression of CD8 T cell responses. PLoS Pathog 10: 1-17, https://doi.org/10.1371/journal.ppat.1004357

Vermeire J, Iannucci V, Naessens E, Van Landeghem K, Vanderstraeten., Van Damme J, Verhasselt B (2013) HIV-1 infection induces a type I IFN responses in primary CD4 $\mathrm{T}$ cells. Retrovirology 10 (Suppl 1): P95. https://doi.org/10.1186/1742-4690-10-S1-P95

White MJ, Kile B (2015) Stressed mitochondria sound the alarm. Immunol Cell Biol 93: 427-428. https://doi.org/10.1038/icb.2015.31

Yan N, Chen ZJ (2012) Intrinsic antiviral immunity. Nat Immunol 13: 214-222. https://doi.org/10.1038/ni.2229

Yesebrant de Lendock L, Martinet V (2014) Interferon regulatory factor 3 in adaptive immune responses. Cell Mol Life Sci 71: 3873-3883. https://doi.org/10.1007/s00018-014-1653-9

Zhao W (2013) Negative regulation of TBK1 - mediated antiviral immunity. FEBS Lett, 587: 542-548. https://doi.org/10.1016/j.febslet.2013.01.052 